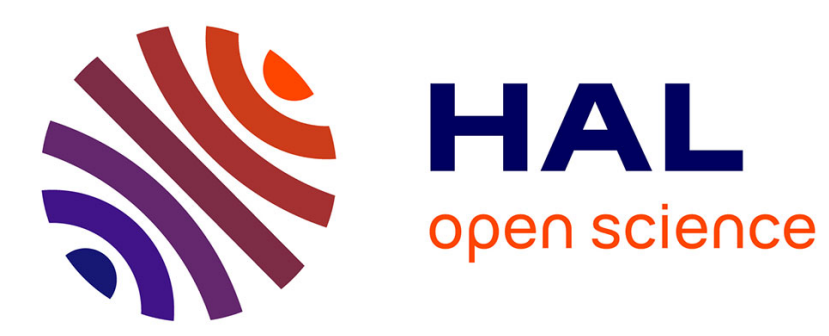

\title{
Actuelles sur les liens entre psychologie individuelle et psychologie sociale \\ Pascale Baligand
}

\section{To cite this version:}

Pascale Baligand. Actuelles sur les liens entre psychologie individuelle et psychologie sociale. Recherches en psychanalyse, 2018, 25 (1), pp.11a. 10.3917/rep1.025.0011a . hal-02523039

\section{HAL Id: hal-02523039 \\ https://hal.science/hal-02523039}

Submitted on 29 Apr 2020

HAL is a multi-disciplinary open access archive for the deposit and dissemination of scientific research documents, whether they are published or not. The documents may come from teaching and research institutions in France or abroad, or from public or private research centers.
L'archive ouverte pluridisciplinaire HAL, est destinée au dépôt et à la diffusion de documents scientifiques de niveau recherche, publiés ou non, émanant des établissements d'enseignement et de recherche français ou étrangers, des laboratoires publics ou privés. 


\title{
Actuelles sur les liens entre psychologie individuelle et psychologie sociale : de l'intérêt de la notion de figure pour penser les discriminations.
}

\begin{abstract}
Résumé :
Dans cet article, l'auteure part d'une recherche collective portant sur l'accès aux soins des patients étrangers ou considérés comme tels pour formuler ses propositions concernant l'apport de la psychanalyse à l'étude des phénomènes de discrimination. Une discussion est ouverte sur le statut de termes survenus lors d'entretiens qualitatifs réalisés auprès de soignants, que la psychologie sociale repère traditionnellement comme des stéréotypes et des préjugés. En s'appuyant sur les travaux de différents auteurs sur la thématique de la présentabilité (Freud, Kahn) et des figures (Lyotard, Didi-Huberman), l'auteure propose de considérer ces termes comme des figures comparables aux figures du travail du rêve, à la fois concrètes et imagées, composites, et mettant en œuvre différentes temporalités individuelles et collectives. Cette démarche permet de souligner l'intérêt de l'étude du registre de la présentation en même temps que celui de la représentation pour aborder la question des discriminations, ainsi que l'intérêt de la notion de figure pour réinterroger les liens entre psychologie individuelle et psychologie sociale avec la psychanalyse.
\end{abstract}

\section{Mots-clés :}

Figure, psychanalyse, stéréotype, discrimination, accès aux soins.

\begin{abstract}
:
Based on a collective project centred on the access to healthcare by foreign patients or patients considered as such, the article proposes to assess psychoanalysis' contribution to the study of discrimination. The author opens a discussion on the terms used by caregivers during qualitative interviews, bearing in mind such terms are traditionally identified by social psychology as stereotypes and prejudices. Drawing on the works of various theorists on the subject of presentability (Freud, Kahn) and figures (Lyotard, Didi-Huberman), the author seeks to consider these terms as figures insofar as they are similar to composite, concrete, and pictorial figures in the dream process (Freud), implementing various individual and collective temporalities. This approach highlights the value of focusing on presentation as well as representation in addressing the question of discriminations, foregrounding the notion of figure to re-examine the links between individual psychology and social psychology with psychoanalytic theory.
\end{abstract}

\section{Key-words :}

Figure, psychoanalysis, stereotype, discrimination, access to healthcare 


\title{
Actuelles sur les liens entre psychologie individuelle et psychologie sociale : de l'intérêt de la notion de figure pour penser les discriminations.
}

\author{
Pascale Baligand
} «Un discours est épais. Il ne signifie pas seulement, il exprime. Et s'il exprime, c’est qu'il a lui aussi du bougé
consigné en lui, du mouvement, de la force, pour soulever la table des significations par un séisme qui fait le
sens ». (Lyotard, 1971, p. 15)

Dans la première partie des Considérations actuelles sur la guerre et la mort, Freud développe les différents aspects de la «désillusion» provoquée par la Première Guerre Mondiale (Freud, 1915b, p. 11). Dans le contexte international conflictuel de l'époque, il constate que l'étranger - notamment du point de vue de la nationalité - se trouve associé à l'hostile, ce qui met à mal certaines croyances présentes dans les sociétés dites civilisées, selon lesquelles les divisions et les violences entre nations auraient été dépassées (Freud, 1915b). Il ajoute que cela tend à se manifester par la résurgence dans le discours social d'un qualificatif particulier pour désigner les membres des peuples considérés comme ennemis : celui de «barbares » (Freud, 1915b, p. 13, p. 16). Le «barbare», celui qui s'exprime en produisant des sons inarticulés, des borborygmes, et qui, ne maniant par une langue, serait incapable de manier la pensée liée à cette langue, est pointé par Freud comme venant incarner en l'occurrence l'étranger de manière privilégiée. Freud propose alors d'interroger l'existence de différents registres de temporalité et de conflit, historiques et psychiques, sociaux et individuels, et les possibles effets d'analogie (Villa, 2016), voire de collusion entre les uns et les autres. Ces réflexions de Freud sont venues entrer en résonnance avec un projet de recherche collectif intitulé «Le malade étranger dans la relation soignant-soigné » (Pestre, Baligand, Wolmark, 2016) ${ }^{1}$, et avec les propositions que j'ai pu développer à partir de ce projet pour contribuer avec la psychanalyse à l'étude des phénomènes de discrimination. Face à des données établies par des recherches récentes en santé publique (Carde, 2007 ; Hamel, Moisy, 2013) et par des recherches menées au sein de différentes associations dont le Comede (Comede, 2014), le projet «Le malade étranger dans la relation soignant-soigné » s'était donné pour but d'apporter un éclairage sur la question de l'accès aux soins des populations étrangères ou considérées comme telles, en mettant l'accent sur l'échelle de la relation soignant-soigné et sur les éventuelles difficultés qui pouvaient émerger dans le cadre de cette relation. Par le biais d'observations et d'entretiens qualitatifs réalisés auprès de soignants de différents services hospitaliers d'Île-de-France, l'ambition était d'appréhender avec la psychanalyse des phénomènes observés à une échelle plus globale par les recherches en santé publique. Lors de ces entretiens, nous avons pu entendre les soignants employer des expressions qui nous ont frappées, et dont je voudrais ici souligner la proximité avec le terme de «barbare» relevé par Freud : les patients étrangers ou considérés comme tels par les soignants ont en effet pu être désignés comme «étrangers avec la barrière de la langue » (on

\footnotetext{
${ }^{1}$ Ce projet a été porté par Elise Pestre (MCU, Université Paris Diderot, UFR Etudes psychanalytiques, Centre de Recherche en Psychanalyse Médecine et Société), en partenariat avec le Comité pour la santé des exilés (Comede), et notamment Laure Wolmark, Responsable du Service de psychothérapie et de santé mentale.
} 
retrouve ici en partie le «barbare »), «déchus socialement», «enfants en détresse », « consanguins», ou encore comme des «Roissy» ou des «HIV». Ces termes, qui ne relèvent pas du même contexte que celui examiné par Freud, mais de la production de modalités de catégorisation, voire d'exclusion, de diverses populations au quotidien, méritaient eux aussi d'être interrogés. Il s'agissait pour nous d'essayer de penser ce qui se jouait pour les soignants au moment où ces expressions leur venaient, et de qualifier plus précisément ce qui émergeait pour eux dans la relation de soin. C'est ici que ce projet collectif a rencontré des thématiques que j'avais eu l'occasion de mettre au travail dans des recherches antérieures (Baligand, 2013), me permettant de développer un certain nombre de propositions. Ces propositions reposent sur une tentative d'élaboration autour de la notion de figure, tant pour penser ce qui se joue au niveau contre-tranférentiel du côté des soignants, que pour mettre en perspective psychologie individuelle et psychologie sociale en lien avec des situations de violence sociale que l'on peut qualifier de discrimination. Le «barbare » ou l' «étranger avec la barrière de la langue », ou encore «l'étranger consanguin », ne pouvaient-ils en effet être pensés comme des figures, notamment en référence aux travaux de Freud sur la prise en considération de la présentabilité (Freud, 1900a)? Pouvait-on les envisager dans leur dimension de présentation plus que de représentation (Kahn, 2001), selon trois aspects propres aux figures du rêve (Freud, 1900a) : leur dimension imagée, leur caractère composite, et leur capacité d'actualisation (au sens de présentation et de mise au présent) d'éléments inconscients? Nous essaierons ici de présenter les perspectives de recherche qui peuvent s'ouvrir pour penser les discriminations à partir de telles propositions. Nous verrons ainsi tout d'abord que cela permet d'élargir l'étude les registres du fonctionnement psychique en jeu dans les phénomènes de discrimination. Nous montrerons ensuite que les dynamiques subjectives à l'œuvre peuvent s'en trouver nouvellement éclairés, avant de revenir sur l'intérêt de la notion de figure pour penser l'articulation entre psychologie individuelle et psychologie sociale.

\section{Représentation et présentation : des stéréotypes aux figures concrètes et imagées.}

Si l'on considère le terme «barbare » relevé par Freud, ou l'expression revenue fréquemment dans les entretiens avec les soignants d' « étranger avec la barrière de la langue », ou encore celle de 1' "étranger déchu socialement", on peut soutenir qu'ils peuvent faire l'objet d'une discussion qui intéresse différents champs de savoir de la psychologie, voire des sciences sociales et humaines de manière plus générale ${ }^{2}$. Dans le champ de la psychologie, ils renvoient traditionnellement à un domaine de recherches bien établi de la psychologie sociale, portant sur les stéréotypes et les préjugés, sur les phénomènes de discrimination associés, ainsi que sur les représentations sociales (Moscovici, 2013). Les stéréotypes, désignant initialement un procédé typographique permettant une impression de masse à faible coût à partir de caractères figés (Amossy, Herschberg-Pierrot, 2014) sont appréhendés en psychologie sociale comme des représentations socialement partagées, rigides et excessivement généralisatrices (Légal, Delouvée, 2015), qui deviennent des préjugés lorsqu'y est adjointe une charge affective particulière. Stéréotypes et préjugés relèvent d'une catégorisation sociale consensuelle, qui s'applique aux membres d'un groupe donné en se focalisant sur leurs traits physiques, de personnalité, ou comportements supposés (Légal, Delouvée, 2015). Ils sont considérés comme pouvant être à la base de comportements

\footnotetext{
${ }^{2}$ On peut penser ici à des travaux de linguistique tels que ceux de Lakoff et Johnson (Lakoff, Johnson, 1980).
} 
discriminatoires, c'est-à-dire à de comportements négatifs sans fondement vis-à-vis des membres de ce groupe (Légal, Delouvée, 2015). Les notions de stéréotypes et de préjugés constituent dans le champ de la psychologie des référentiels majeurs pour aborder la question des inégalités de traitement fondées sur des critères arbitraires tels que l'appartenance à un groupe, le genre, la race ou la nationalité (Whitley, Kite, 2013). Les termes d' «étranger avec la barrière de la langue » rappelant le «barbare », ou encore celui d' «étranger déchu socialement », pourraient dans ce cadre être considérés comme des stéréotypes ayant trait à une catégorie de personnes donnée : les patients «étrangers ». Associés pendant les entretiens à des situations de violence sociale dépassant le cadre de la relation soignant-soigné, mais dont on peut supposer qu'elles l'affectent et qu'il faut en tenir compte dans le soin (TummalaNarra, 2015), ainsi qu'à des situations professionnelles considérées par les soignants comme particulièrement complexes et difficiles, ces termes pourraient apparaître comme des exemplaires de la catégorie stéréotypée du «patient étranger», ou comme des éléments relevant de son champ d'extension.

On peut cependant remarquer qu'au-delà de leur relative rigidité ou de leur caractère socialement partagé, ces termes peuvent être porteurs de traits plus singuliers, qu'il peut être intéressant de déplier. Au-delà de la question du champ disciplinaire convoqué pour l'étude des phénomènes de discrimination, c'est alors aussi celle des registres du fonctionnement psychique en jeu dans ces situations qu'il convient de soulever. On peut d'ailleurs interpréter dans ce sens les recherches récentes en psychologie sociale, qui mettent davantage l'accent sur une dimension automatique du fonctionnement psychique à l'œuvre dans la production des stéréotypes et des préjugés (Ogunnaike, Dunham, \& Banaji, 2010), et s'interrogent sur les associations qui s'établissent implicitement entre telle ou telle représentation (Greenwald, Banaji, Rudman, Farnham, Nosek \& Mellott, 2002 ; Carney, Krieger \& Banaji, 2010). Ces recherches expérimentales convoquent une dimension implicite du psychisme, dont on pourrait se demander les liens qu'elle entretient avec une dimension inconsciente telle que la psychanalyse la conçoit. A partir d'une modalité d'écoute des entretiens réalisés référée à l'écoute psychanalytique (Kahn, 2012) et incluant une prise en compte de la dimension sociale des situations étudiées (Jodelet, 1989), on peut même soutenir que les termes employés par les soignants lors des entretiens sollicitent, peut-être davantage que le registre des représentations consensuelles et figées - au sens des stéréotypes décrits par la psychologie sociale, mais peut-être même des rêves typiques décrits par Freud (Freud 1900a) -, une forme de «pensée en image » (Barreau, 2017, p. 197), un registre du fonctionnement psychique et de la parole où la présentation (Freud, 1900a ; Kahn, 2001) et l'expression figurale (Lyotard, 1971 ; Schefer, 1999) prédomineraient sur la signification. Cela rappellerait ce qui a été mis au jour par Freud à partir de l'étude des processus de rêve et de la formation de symptôme (Freud, 1895d). Au cours des entretiens, les expressions auxquelles ont pu recourir les soignants nous ont en effet frappées par leur caractère concret et imagé, et par leur forte présence sensorielle. Ainsi, dans les situations où émergeaient des difficultés de compréhension linguistique, les professionnels interviewés évoquaient fréquemment «des patients étrangers dans le service, où il y a la barrière de la langue comme frontière ", ou encore des patients avec qui il est nécessaire d'avoir recours « aux gestes », aux «bruitages », « aux dessins », engageant ainsi une forme de dialogue de type régressif. Le recours à cette expression de la «barrière de la langue » permettait aux soignants de signifier, lors de l'interview, la difficulté, voire l'impossibilité de prodiguer des soins à tel ou tel patient. Cette formulation imagée venait ainsi donner à voir un obstacle comme matériellement éprouvé et perçu dans l'actualité de la relation de soin. L' "étranger déchu socialement », chez qui un soignant suspectait la présence d'une dépression latente, venait quant à lui mettre en scène au sens littéral du terme la figure d'un patient tombé, traversant un moment d'effondrement 
psychique et matériel, et qu'il faudrait recueillir. Le «barbare» pointé par Freud convoque d'ailleurs lui aussi une dimension sensorielle très forte, puisque ce terme a été construit avec pour principe même de donner à entendre les sons inarticulés que le sujet qui tente d'établir un lien avec l'autre n'arrive pas à entendre et à ordonner. La dimension concrète, sensorielle et imagée qui est repérable dans ces expressions relève du registre de la présentation davantage que de celui de la représentation, ce qui permet de les qualifier de «figures », au sens de Darstellungen (Kahn, 2001). Ces figures convoquent une forme de pensée en image (Barreau, 2017), de tendance à partir de formulations verbales abstraites pour les échanger contre des formulations plus imagées, caractéristiques pour Freud du travail du rêve (Freud, 1900a) et dont le but est de contourner la censure pour faire place de manière déguisée à des éléments refoulés. Ce procédé, appelé prise en considération de la présentabilité, va conférer au contenu du rêve son caractère visuel, en jouant notamment sur la polysémie et l'équivocité ${ }^{3}$, et donner l'impression au rêveur qu'il vit telle ou telle situation au présent. On pourrait faire l'hypothèse que c'est ce même procédé qui a cours dans les entretiens avec les soignants lorsqu'ils évoquent leur pratique et qu'ils associent telle ou telle relation de soin ou tel ou tel moment à des éléments d'étrangeté chez le patient. Face à un patient ou à une situation convoquant des éprouvés contre-tranférentiels particuliers, l'empêchement et l'étrangeté dans le lien, produit d'un effet de collusion avec des éléments inconscients présents chez le soignant, seraient ainsi sensoriellement mis en scène, et trouveraient en telle ou telle figure une voie d'expression concrète et imagée. Freud reliant le caractère visuel et sensoriel du rêve au processus régrédient (Botella, 2001) et à un retour à des éléments perceptifs précédant la représentation, on pourrait se demander si les stéréotypes, définis par leur fixité, pourraient être considérés comme des formes de fixation des représentations sociales dans des contextes conflictuels socialement et psychiquement, là où l'apparition de figures relèverait de processus de régression aboutissant au recours à des termes imagés.

\section{Des figures composites mettant en jeu le narcissisme du sujet}

Un autre trait caractéristique de ces figures qui sont apparues lors des entretiens avec les soignants rappelle lui aussi la dimension plastique du travail du rêve, et les autres facteurs de ce travail du rêve que Freud identifie, à savoir la condensation et le déplacement (Freud, 1900a). Certains termes relatifs aux patients perçus comme étrangers qui ont émergé dans les entretiens semblaient en effet porter la marque de la mise en œuvre de ces procédés. Les patients perçus comme étrangers étaient régulièrement présentés sous la forme de figures condensant plusieurs traits, parfois à la limite du fantastique, que les soignants n'arrivaient pas à déconstruire et sur lesquelles semblaient se heurtaient leurs connaissances et leurs habitudes professionnelles. Un professionnel relatait ainsi le cas d'une patiente étrangère à l'hôpital avec qui il avait «essay[é] de savoir ce qui se pass[ait] » car, disait-il, "l'histoire n'arrive pas avec [le patient], il faut détricoter ce qui s'est passé avant ». En quête de sens, cette tentative de «détricotage» pouvait rappeler les associations libres par lesquelles

\footnotetext{
${ }^{3}$ Freud donne l'exemple d'une haute tour présente dans le rêve d'une patiente (Freud, 1900a), qui se révèle être un moyen d'inclure dans ce rêve un personnage précis lié au souhait inconscient de la rêveuse, car cette haute tour vient figurer une personne «grande comme une tour » et conjointement enfermée dans un asile, désigné en allemand par la «tour des fous ». Ici, le recours à la polysémie et à la possibilité d'utiliser le terme « tour» au sens figuré en fait un élément privilégié par le rêveur qui cherche à exprimer une pensée de rêve de manière déguisée. On pourrait également trouver de nombreux exemples mettant en jeu la prise en considération de la présentabilité dans la formation de symptôme, fondés eux-aussi sur l'équivocité entre sens propre et sens figuré (cf. par exemple «alleinstehen », dans le cas de Elisabeth von R. (Freud, 1895d).
} 
l'analyse va pouvoir décomposer les figures condensées du rêve (comme le personnage composite d'Irma, ou du rêve de l'oncle à la barbe jaune, (Freud, 1900a)) ou les identifications condensées qui contribuent à la formation du symptôme (comme la toux hystérique de Dora, (Freud, 1905e)). La production de ces figures composites à partir de la condensation et du déplacement reposerait là aussi sur l'élection inconsciente de tel ou tel trait présent chez le patient (précarité, tristesse, situation de victime, etc.) et par l'identification à ce trait de la part du soignant, en particulier dans la mesure où ce trait en rappelle un autre qui fait l'objet d'un refoulement. Tout comme dans la formation de symptôme, certains traits du patient pourraient être choisis inconsciemment pour leur capacité à incarner conjointement ou de manière allusive et déplacée des représentations refoulées et des traits du moi du soignant. La figure du «patient enfant », souvent présente dans les paroles des soignants, témoigne bien de cette complexité de la composition figurale fondée sur la condensation. Elle convoque aussi bien une facette du moi du soignant qui a trait à la sexualité infantile, qu'une autre facette venant rappeler le nourrisson en situation de détresse, correspondant dans les interviews à l'évocation de patients traversant des moments de désœuvrement intense, suscitant ou actualisant des sentiments archaïques chez les soignants.

Rappelons que Freud soutient que les éléments du rêve, y compris les personnages différents du rêveur, sont porteurs du narcissisme du sujet (Freud, 1900a). Lacan, à la suite d'une discussion sur la décomposition du personnage d'Irma dans le rêve paradigmatique rapporté par Freud, souligne les enjeux sous-jacents à la question de l'identification telle qu'elle se pose dans le rêve. Il estime alors que le rêve est le théâtre de l'apparition de la « série des moi $\gg^{4}$ du rêveur (Lacan, 1954-1955, p. 228). On peut supposer que lorsqu'un élément issu de problématiques intimes frappées de refoulement fait retour pour le soignant dans le cadre de la relation de soin, la possibilité pour lui de conserver une flexibilité dans le lien se trouverait compromise. Le patient serait perçu comme avant tout étranger car il viendrait incarner une facette du moi du soignant, de l'ordre de l'étrangement inquiétant (Freud, 1919h), voire de l'irreprésentable, la figure devenant ici proche d'une figure du réel au sens où Ginette Michaud l'a développé (Michaud, 1999). Paradoxalement, c'est en raison d'une proximité très forte demeurée inconsciente et renvoyant à des éléments refoulés ou clivés que s'instaurerait avec tel ou tel patient une relation spécifique, où le patient deviendrait un support d'expression de ces éléments. Tout se passe comme si la «série des moi » de tel ou tel soignant trouvait dans une relation donnée, en lien avec une problématique demeurée inconsciente pour lui, des voies de présentation et des modalités de dramatisation à travers la construction subjective de figures de l'étranger. Dans le contexte d'une situation à traiter particulièrement difficile, c'est aussi parfois le caractère extrême des figures de l'étranger convoquées par les soignants qui retient l'attention, tant certains traits auxquels ils s'identifient vont exercer sur eux des effets de captation, voire de sidération. Cela peut même aller jusqu'à l'expression d'un trait fortement investi de manière directe, comme si l'angoisse était telle pour le soignant qu'elle s'exprimait de manière crue, sans qu'il puisse y avoir un déplacement sur un trait neutre ou moins investi. On remarque alors que l'élection inconsciente d'un trait unique, aussi fortement investi, peut produire une figure de l'étranger réduite à ce trait, la fixité et la rigidité semblant prendre le dessus dans la relation de soin. Cela est par exemple le cas avec certaines figures telles que « Roissy » ou encore «HIV ». La notion même de figure peut d'ailleurs ici être mise à la discussion, tant le caractère rigide semble laisser peu de place à la plasticité que suppose la prise en considération de la présentabilité. En tout état de cause, la possibilité d'entendre dans la parole de l'interviewé plusieurs registres de ce qui se joue dans la relation de soin semble un point d'ouverture pour

\footnotetext{
${ }^{4}$ Lacan J., Le moi dans la théorie de Freud et dans la technique de la psychanalyse, Seuil, 1954-1955, 1981, p. 228.
} 
approfondir la compréhension des phénomènes à l'œuvre dans des situations mettant en jeu des formes de violence sociale.

Temporalités individuelles et temporalités sociales. Figures et « revenances fantômales ».

Une troisième dimension caractérisant les figures de l'étranger apparues lors des entretiens avec les soignants permet en dernier lieu de revenir sur l'articulation entre psychologie individuelle et psychologie sociale, notamment pour ce qui concerne les temporalités à l'œuvre pour le sujet et dans le collectif. En plus de leur dimension imagée, plastique et composite, les figures pourraient en effet se caractériser par leur potentialité d'actualisation de différents registres temporels. Comme nous avons commencé à l'évoquer avec la question du refoulement qui se joue au niveau individuel pour chaque soignant, les figures repérées lors des entretiens semblent pouvoir combiner différents registres temporels. A cet égard, certaines figures sont particulièrement intéressantes, dans la mesure où elles combinent une temporalité psychique individuelle où la linéarité a été abolie du fait de la persistance d'éléments refoulés demeurés actuels avec une temporalité collective, qui pourrait elle aussi fonctionner sur un mode non linéaire. C'est notamment le cas de la figure du «patient enfant ». On se trouverait ici face non seulement à des résurgences de l'infantile du soignant, où l'on pourrait repérer des imagos parentales ou des fantasmes liés à la sexualité infantile, mais aussi face à des traces que l'on pourrait considérer comme issues de l'histoire commune, en l'occurrence coloniale, produisant des effets d'anachronisme saisissants. Si Freud a affirmé que «toute psychologie individuelle est aussi et en même temps une psychologie sociale » (Freud, 1921c, p. 137) et a ouvert ainsi de multiples voies de recherches en sciences humaines (Haber, 2012), c'est peut-être aujourd'hui la question de la forme de ces liens entre subjectif et commun, des mécanismes en jeu dans les effets de temporalité qui marquent la vie individuelle et collective, et des échelles d'interprétation que l'on choisit, qui peut ouvrir des prolongements de recherches en psychanalyse, en particulier sur les thématiques liées aux discriminations. Freud n'a cessé de souligner les particularités de la temporalité à l'œuvre dans l'inconscient, et d'en proposer des métaphores (Freud, 1896) et des modélisations (Freud, 1896 ; Freud, 1900a). De nombreux travaux produits dans le champ de la psychanalyse ont par ailleurs montré le caractère fondamental pour le sujet et pour le traitement du traumatisme individuel de la possibilité d'une élaboration collective de certains événements de l'histoire (Altounian, 2000 ; Waintrater, 2003). Avec les figures que les interviewés ont employées, tout comme avec l'utilisation du terme de «barbare » repérée par Freud dans la société de son temps, on pourrait s'interroger sur la persistance et le traitement à l'échelle individuelle d'éléments faisant référence à des événements traumatiques ou non encore traités communs aux membres d'un groupe. La possibilité de mettre ainsi en lumière des effets de temporalité fonctionnant dans le collectif à l'image ou parfois en concordance de ceux de l'inconscient tels qu'il se déploie dans le psychisme individuel se révèlerait pertinente pour réexaminer avec la psychanalyse les liens entre psychologie individuelle et psychologie sociale. Les travaux de Didi-Huberman sur l'histoire de l'art offrent en l'occurrence une possibilité pour articuler des figures produites à l'échelle du sujet, mais qui le seraient comme en écho avec des figures socialement transmises. Didi-Huberman affirme en effet que certaines œuvres d'art se présentent telles «un noeud de temps difficile à déchiffrer parce que s'y recroisent sans cesse des mouvements d'évolution et des mouvements qui résistent à l'évolution » (DidiHuberman, 2002, p. 53). Cherchant à souligner la pertinence d'une conception de l'histoire de l'art dans laquelle des effets de temporalités particuliers seraient pris en compte, il propose d'analyser les images et les œuvres d'art à partir de formes de persistance et de retour traversant les époques et les courants, et qu'il qualifie de «revenance fantômale » (DidiHuberman, 2002, p. 41). Ses propositions permettent de penser une dimension de présentation 
et de présentabilité œuvrant à l'échelle collective, et peuvent amener à considérer les figures qui ont émergé lors des entretiens avec les soignants comme des « survivances fantomales » (Didi-Huberman, 2002, p. 41), traces d'un passé commun dont les effets de retour ne seraient pas linéaires mais s'actualiseraient par l'apparition ou la réapparition de traits sur certaines figures singulières. Les différentes figures de l'étranger repérées lors des entretiens évoquent alors cette «revenance fantômale» de traces du passé, où affleurent tant des éléments fantasmatiques, propres au soignant, que des éléments de l'histoire commune. La notion de figure, notamment telle qu'éclairée par des figures comme celle du «patient enfant», viendrait alors éclairer singulièrement l'intrication entre psychologie individuelle et psychologie sociale, et les analogies ou collusions entre temporalité inconsciente du sujet et temporalité collective, notamment par le biais d'effets de « revenance fantômale ».

\section{Conclusion :}

La recherche collective «Le malade étranger dans la relation soignant soigné » a permis de repérer l'usage par les soignants d'un certain nombre de termes qui mettaient en jeu des éléments issus de l'imaginaire collectif, des expressions littérales du sens commun, ou encore des composantes plus singulières, propres à telle relation entre tel soignant et tel patient. La survenue de ces termes, notamment en lien avec l'évocation par les soignants de moments difficiles dans les prises en charge, a pu être considérée comme le fruit d'un travail psychique complexe, à entendre selon différents registres propres à l'écoute analytique, et que j'ai proposé de penser à partir de différents travaux portant sur la présentabilité et sur la notion de figure, issus du champ de la psychanalyse ou de champs connexes qui sont amenés à l'interroger. Il s'agissait ici d'entendre dans les entretiens ce qui pouvait apparaître et s'exprimer sur le registre de la présentation davantage que sur celui de la représentation, et d'élargir ce faisant la palette des outils d'analyse des obstacles à l'accès aux soins des personnes étrangères ou considérées comme telles, et plus généralement des phénomènes de discrimination. Dans une perspective scientifique et épistémologique, les dimensions caractéristiques des figures apparues lors des entretiens (concrètes et imagées, composites et actualisant différents registre temporels) rendent compte de la notion de figure en tant qu'elle permet d'ouvrir une discussion autour de celles de stéréotype et préjugé telles qu'étudiées en psychologie sociale. Les figures, pensées selon les caractéristiques évoquées, seraient en effet susceptibles d'être liées à des représentations générales, voire typiques, mais de désigner également des potentialités plastiques que chaque sujet pourrait mettre en œuvre à des fins inconscientes singulières. Elles permettraient également de penser les temporalités à l'œuvre à l'échelle collective, et d'envisager à cette échelle des formes et des modalités de persistance de traces du passé qui ne seraient pas encore représentées et intégrées. Ajoutons enfin qu'au plan clinique, la prise en compte des liens entre la survenue de ces figures et l'évocation de prises en charge considérées comme particulièrement complexes par les soignants, avec des effets de court-circuit ou des difficultés pour les professionnels à effectuer leurs tâches habituelles, pourraient se révéler pertinentes dans le cadre de dispositifs d'analyse des pratiques.

\section{Bibliographie}


Altounian, J. (2000), La survivance. Traduire le trauma collectif. Paris : Dunod, 2000.

Amossy, R., Herschberg-Pierrot, A. (2011), Stéréotypes et clichés. $3^{\text {ème }}$ édition. Paris : Armand Colin, 2014.

Baligand, P., (2013), Une chambre à soi. Étude psychanalytique de la notion de chez-soi à partir des liens entre espace et présentabilité. Thèse de doctorat sous la direction du Professeur Patrick Guyomard, Université Paris 7.

Barreau, J.-J. (2017), Qu'appelle-t-on penser... en image ? Dans Barreau, J.-J. (dir.), Actes du Quatrième Groupe. Le rêve, entre actuel et origines (p. 197-272). Paris : In Press, 2017.

Botella, C. (2001), Figurabilité et régrédience. Revue française de psychanalyse, ${ }^{\circ} 4$, vol. 65, p. 1149-1239. DOI 10.3917/rfp.654.1149.

Carde, E. (2007), Les discriminations selon l'origine dans l'accès aux soins, Santé publique, vol. 19, n 2, p. 99-109. DOI 10.3917/spub.072.0099.

Carney, D., Krieger, N., \& Banaji, M. R. (2010), Implicit measures reveal evidence of personal discrimination. Self and identity, 9(2), p. 162-176. DOI : $10.1080 / 13594320902847927$.

Comede (comité médical pour les exilés), (2014), Rapport 2014, http://www.comede.org/IMG/pdf/RapportComede2014.pdf.

Didi-Hüberman, G., L'image survivante. Histoire de l'art et temps des fantômes selon Aby Warburg. Paris : Editions de Minuit, 2002.

Freud, S., Breuer J. (1895d), Études sur l'hystérie [Studien über hysterie. GW I], Traducteur : Berman, A., Paris : PUF, 2007.

Freud, S. (1900a), L'interprétation du rêve, OCF IV [Traumdeutung, GW II-III]. Traducteurs : Altounian, J., Cotet, P., Lainé, R., Rauzy, A., Robert, F., Paris : PUF, 2004.

Freud, S. (1905e), Fragment d'une analyse d'hystérie (Dora). Cinq psychanalyses [Bruchstück einer Hysterienanalyse. GW V]. Traducteurs : Bonaparte, M., Loewestein, R. Paris : PUF, 2006.

Freud, S. (1915b), Considérations actuelles sur la guerre et la mort [Zeitgemässes über Krieg und Tod, GW X]. Traducteurs : Cotet, P., Bourguignon, A., Cherki, A., Paris : Payot, 2001.

Freud, S. (1919h), L'inquiétante étrangeté [Das Unheimliche, GW XII], dans L'inquiétante étrangeté et autres essais (p. 209-263). Traducteur : Féron, B., Paris : Gallimard, 1985.

Freud, S. (1921c), Psychologie des foules et analyse du moi [Massenpsychologie und IchAnalyse, GW XIII ]. Traducteurs : Cotet, P., Bourguignon, A., Cherki, A., Paris : Payot, 2001.

Freud, S. (1950c), La naissance de la psychanalyse. Lettres à Wilhelm Fliess. Notes et plans (1887-1902). Traducteur : Berman, A. Paris : PUF, 2009.

Greenwald, A. G., Banaji, M. R., Rudman, L., Farnham, S., Nosek, B. A., \& Mellott, D. (2002), A unified theory of implicit attitudes, stereotypes, self-esteem and self concept. Psychological Review, 109, 1, p. 3-25. DOI: 10.1037//0033-295X.109.1.3.

Haber, S. (2012), Freud et la théorie sociale. Paris : La Dispute, 2012. 
Hamel, C., Moisy M. (2013), Immigrés et descendants d'immigrés face à la santé. Documents de travail, $\mathrm{n}^{\circ}$ 190, Paris : INED, 2013.

Jodelet, D. (dir.), (1989), Les représentations sociales. Paris : PUF, 2003.

Kahn, L. (2001), L’action de la forme. Revue française de psychanalyse, vol. 65, n 4, p. 983 1056. DOI 10.3917/rfp.654.0983.

Kahn, L. (2012), L'écoute de l'analyste. De l'acte à la forme. Paris : PUF, 2012.

Lacan J. (1954-1955), Le moi dans la théorie de Freud et dans la technique de la psychanalyse, Paris : Seuil, 1981.

Lakoff G., Jonhson M. (1980), Metaphors we live by. Chicago : The University of Chicago Press, 2003.

Légal, J.-B., Delouvée, S. (2008), Stéréotypes, préjugés et discrimination, $2^{\text {ème }}$ édition. Paris : Dunod, 2015.

Lyotard, F. (1971), Discours, figure. Paris : Klincksieck, 2002.

Michaud, G. (1999), Figures du réel, Paris : Denoël, 1999.

Moscovici, S. (2013), Le scandale de la pensée sociale, Paris : Éditions de l’EHESS, 2013.

Ogunnaike, O., Dunham, Y., \& Banaji, M. R. (2010), The language of implicit preferences. Journal of Experimental Social Psychology, 46(6), p. 999-1003. DOI : 10.1016/j.jesp.2010.07.006.

Pestre, E., Baligand, P., Wolmark, L., (2016), Ségrégations à l'hôpital. De quelques figures de l'étranger dans la relation de soin. Cliniques Méditerranéennes, $\mathrm{n}^{\circ}$ 94, p. 51-67. DOI 10.3917/cm.094.0051.

Schefer, O. (1999), Qu'est-ce que le figural ? Critique, nº 630, p. 912-925.

Tummala-Narra, U. (2015), Cultural competence as a core emphasis of psychoanalytic theory. Psychoanalytic Psychology, 32(2), p. 275-292. DOI : 10.1037/a0034041.

Villa, F. (2016), Totem et tabou: une méthode pour l'interdisciplinarité ? Recherches en psychanalyse, $\mathrm{n}^{\circ} 21$, p. 9-20. http://www.cairn.info/revue-research-in-psychoanalysis-2016-1page-9a.htm.

Waintrater, R. (2003), Sortir du génocide. Témoigner pour réapprendre à vivre. Paris : Payot, 2003.

Whitley, B. E. \& Kite, M. E. (2006), Psychologie des préjugés et des discriminations. [The Psychology of Prejudice and Discrimination]. Traducteur: Arciszewski, T., Bruxelles : De Boeck, 2013. 
